\title{
Coupled 3-D Finite Difference Time Domain and Finite Volume Methods for Solving Microwave Heating in Porous Media
}

\author{
Duško D. Dinčov ${ }^{1}$, Kevin A. Parrott ${ }^{1}$, and Koulis A. Pericleous ${ }^{1}$ \\ University of Greenwich, \\ School of Computing and Mathematical Sciences, \\ 30 Park Row \\ London SE10 9LS \\ \{D.Dincov, A.K.Parrott, K.Pericleous\}@gre.ac.uk \\ http://cms1.gre.ac.uk
}

\begin{abstract}
Computational results for the microwave heating of a porous material are presented in this paper. Combined finite difference time domain and finite volume methods were used to solve equations that describe the electromagnetic field and heat and mass transfer in porous media. The coupling between the two schemes is through a change in dielectric properties which were assumed to be dependent both on temperature and moisture content. The model was able to reflect the evolution of temperature and moisture fields as the moisture in the porous medium evaporates. Moisture movement results from internal pressure gradients produced by the internal heating and phase change.
\end{abstract}

\section{Introduction}

Microwaving is a common means of heating foodstuffs as well as an important industrial process for heating water-based materials and removing moisture from porous materials such as the drying of textiles, wood, paper and ceramics. Perhaps the largest consumer of microwave power is the food industry where it is used for cooking, thawing, pasteurization, sterilization etc. The ability of microwave radiation to penetrate and interact with materials provides a basis for obtaining controlled and precise heating. Although microwave heating is most beneficial when used for materials with a high moisture content, other materials can still be heated efficiently and quickly.

Because of dielectric losses, microwave absorption provides a volumetrically distributed source. The temperature and moisture distributions in a material during microwave heating are influenced by the interaction and absorption of radiation by the medium and the accompanying transport processes due to the dissipation of electrical energy into heat.

Dielectric properties of most biomaterials vary with temperature [1]. Torres at al. proposed in [11] a 3-D algorithm for microwave heating by coupling of the power distribution with the heat transfer equations in both frequency and 
temperature dependent media. The model was able to predict the locations of hot spots within the material. The transfer of mass was assumed to be unimportant.

However, if significant drying occurs during heating, mass transfer must be accounted for too. An one dimensional multiphase porous media model for predicting moisture transport during intensive microwave heating of wet biomaterials was proposed in [8]. The transport properties varied with structure, moisture and temperature. The model successfully predicted the moisture movement. Excellent research into drying of wood was carried out by Perre [9]. Their 3-D algorithms coupled heat and mass transfer with Maxwell's equations and the dielectric properties depended both on temperature and moisture.

The present work considers microwave heating of a simple saturated porous material with temperature and moisture dependent dielectric properties. The solution of Maxwell's equations is performed in the time domain using the Finite Difference Time Domain technique. The 3-D heat and mass transfer equations are solved using the Finite Volume code PHOENICS [10]. Full coupling between the two calculations is achieved by mapping the (moisture and temperature dependent) porous media dielectric properties from the CFD mesh onto the electromagnetic solver and then mapping the microwave heating function from the finite difference mesh to the finite volume mesh.

\section{Electomagnetic field in microwave enclosures}

The distribution of electromagnetic field in space and time is governed by Maxwell's equations [13]. When material interfaces are present, boundary conditions must be imposed to account for discontinuities of charge and current densities. Provided both media have finite conductivity and there are no sources at the interface, the tangential electric and magnetic fields along the interface are continuous.

\subsection{Dielectric Properties}

The dielectric properties of materials subjected to microwave heating play a key role in designing proper microwave applicators. They are: permittivity $\epsilon$, permeability $\mu$ and conductivity $\sigma$. Permittivity describes the interaction of the material with the high frequency electric field and is defined by the following equation :

$$
\epsilon=\epsilon_{0}\left(\epsilon_{r}^{\prime}-\epsilon_{e f f}^{\prime \prime}\right)
$$

where $\epsilon_{r}^{\prime}$ is the relative dielectric constant, $\epsilon_{e f f}^{\prime \prime}$ is the effective relative loss factor and $\epsilon_{0}=8.85 \times 10^{12} \mathrm{~F} / \mathrm{m}$ is the permittivity of air. $\epsilon_{r}^{\prime}$ is a measure of the polarizability of a material in the electric field, and $\epsilon_{\text {eff }}^{\prime \prime}$ includes the loss factors which are relevant to high frequency heating.

Both $\epsilon_{r}^{\prime}$ and $\epsilon_{\text {eff }}^{\prime \prime}$ are temperature $(T)$ dependent and a number of investigations have been made in order to explain this behaviour [1],[5]. In most cases, 
their values increase as the material thaws and thereafter decrease as the temperature increases. However, these changes for the temperatures up to the boiling point are relatively small [5].

Since most foodstuffs contain an appreciable amount of water, the variation of $\epsilon_{r}^{\prime}$ and $\epsilon_{e f f}^{\prime \prime}$ with the moisture content plays an important role in the design of the microwave heating process. If the temperature dependence in known, the relative dielectric constant and the electric permittivity can be averaged using the following representation (see e.g. [9]):

$$
\bar{\epsilon}(M, T)=(1-\phi) \epsilon^{\text {solid }}(T)+\phi\left(M \epsilon^{\text {liquid }}(T)+(1-M) \epsilon^{\text {gas }}(T)\right)
$$

where $M$ is a moisture content and $\phi$ porosity.

Losses under the influence of the magnetic field can be described in a similar way to losses in electric materials. However, most materials used in microwave processing are magnetically transparent. The magnetic permeability in this work is assumed to have the value of the free space permeability $\mu_{0}=4 \pi \times 10^{-7} \mathrm{H} / \mathrm{m}$.

\subsection{Finite-Difference Time-Domain (FTDT) Scheme}

Yee's scheme [13] is used to discretize the Maxwell's equations. The FTDT Scheme proceeds by segmenting the volume into a three-dimensional mesh composed of a number of finite volumes.

It makes use of finite difference approximations to electric and magnetic fields components, that are staggered both in time and space. $E$ and $H$ field components are positioned at half-step intervals around unit volumes and they are evaluated at alternate half-time steps, effectively giving centered difference expressions for both time and space derivatives.

Values for $\epsilon$ and $\sigma$ are specified at cell centres as $\epsilon(i, j, k)$ and $\sigma(i, j, k)$. Continuity across the interface of the tangential field is implemented automatically.

This scheme is second order accurate in both time and space on uniform and non-uniform meshes [7], and can be locally refined [6] without significant loss of accuracy. The use of (semi)-structured meshes ensures optimally fast computations for the most time-consuming component of the overall calculations and is sufficient for our geometric modelling requirements.

\section{Heat and Mass Transfer in Porous Media}

\subsection{Dissipated Power}

The dissipated power density is the microwave energy absorbed by the material. It is eventually converted into thermal energy. The dissipated power density is influenced by the field intensity distribution and electric properties. The heating, $Q$, which will be included as a source term in the heat transfer equation, is computed from peak field amplitudes as:

$$
Q=\frac{1}{2}\left|E_{\max }\right|^{2} .
$$




\subsection{Heat Transfer Equations}

There are two energy transfer equations within the processed specimen each corresponding to one "phase". The first phase consists of solid and liquid and the second one is the gas phase.

$$
\begin{gathered}
\bar{\rho}_{1} \frac{\partial H_{1}(x, y, z, t)}{\partial t}+\nabla \cdot\left(\bar{\rho}_{1} \mathbf{u}_{1} H_{1}-\frac{\bar{k}_{1}}{\bar{C}_{p 1}} \nabla H_{1}(x, y, z, t)\right)=Q(x, y, z, t)+S_{1}^{i n t} \\
\bar{\rho}_{2} \frac{\partial H_{2}(x, y, z, t)}{\partial t}+\nabla \cdot\left(\bar{\rho}_{2} \mathbf{u}_{2} H_{2}-\frac{\bar{k}_{2}}{\bar{C}_{p 2}} \nabla H_{2}(x, y, z, t)\right)=S_{2}^{\text {int }}
\end{gathered}
$$

where the specific heat, $C_{p}$ is averaged for each phase as follows:

$$
\begin{gathered}
\bar{C}_{p 1}=(1-\phi) C_{p 1}^{\text {solid }}+\phi M C_{p 1}^{\text {liquid }} \\
\bar{C}_{p 2}=\phi(1-M) C_{p 2}^{\text {gas }}
\end{gathered}
$$

Thermal conductivity $k$ and density $\rho$ are averaged in a similar way. The heating function is included in the solid-liquid heat transfer equation. There will be an interphase transfer between the two phases, represents by the interface source $S_{i}^{\text {int }}$ defined as:

$$
S_{i}^{i n t}=h_{i j} A_{s}\left(H_{i}^{i n t}-H_{i}\right)
$$

where $h_{i j}$ is a bulk-to interface heat transfer coefficient, $A_{s}$ is the total interface area and $H^{\text {int }}$ are interface enthalpies.

\subsection{Mass transfer equations}

Gas phase continuity equation:

$$
\frac{\partial\left(\rho_{g} r_{g}\right)}{\partial t}+\nabla \cdot\left(\rho_{g} r_{g} \mathbf{u}_{g}\right)=\dot{m} .
$$

Liquid phase continuity equation:

$$
\frac{\partial\left(\rho_{l} r_{l}\right)}{\partial t}+\nabla \cdot\left(\rho_{l} r_{l} \mathbf{u}_{l}\right)+\dot{m}=0 .
$$

$r_{g}$ and $r_{l}$ are gas and liquid volume fractions respectively. The interface mass transfer rate, $\dot{m}$, is determined from the balance of heat through the interface between the phases.

\subsection{Darcy's Law Equations}

Darcy's law can be used to represent momentum transfer in the porous medium. For liquid it can be expressed in the form:

$$
\mathbf{u}_{l}=-\frac{K_{l}}{\lambda_{l}}\left(\nabla P_{g}+l_{r} \nabla r_{l}+l_{T} \nabla T\right)
$$


in which $l_{r}=-\partial P_{c}\left(r_{l}, T\right) / \partial r_{l}$ and $l_{T}=-\partial P_{c}\left(r_{l}, T\right) / \partial T$ are terms related to capillary pressure $P_{c}$. Also $K_{l}$ and $\lambda_{l}$ denote the permeability tensor and the liquid viscosity, respectively, and $P_{g}$ gas pressure.

The appropriate version of Darcy's law for the gas phase is

$$
\mathbf{u}_{g}=-\frac{K_{g}}{\lambda_{g}}\left(\nabla P_{g}\right)
$$

where $K_{g}$ and $\lambda_{g}$ are the permeability tensor and the liquid viscosity, respectively.

\subsection{Initial and Boundary Conditions}

There are number of surface heat or surface loss mechanisms during the microwave heating. The general boundary condition on the material's surface can be expressed as:

$$
-k \frac{\partial T}{\partial n}=h_{c}\left(T_{s}-T_{\infty}\right)+\sigma_{r a d} \epsilon_{r a d}\left(T_{s}^{4}-T_{\infty}^{4}\right)-\dot{m} L
$$

where $T_{s}$ is the load surface temperature, $T_{\infty}$ is the convective air temperature, $n$ represents the normal to the surface, $h_{c}$ is the convective heat transfer coefficient, $\sigma_{\text {rad }}$ is the Stefan-Boltzmann constant, $\epsilon_{\text {rad }}$ is the radiative surface emissivity and $L$ is the latent heat of vaporization of the evaporated liquid. The first term in the equation represent the natural convection by which the load is cooled. The second term is the surface radiation and is important as a cooling mechanism at high load temperatures, or as a heating mechanism if susceptors are used. Since materials with a high moisture content are being observed in this study, the last term, evaporative cooling will have the strongest impact on the temperature profile.

Besides, initial conditions for the example considered here also included:

$$
P_{g}(t=0)=P_{a t m}, T(t=0)=T_{\text {ambient }} .
$$

\section{Results and Discussion}

\subsection{Model Description}

The microwave oven model used in this paper consists of a waveguide and a microwave cavity containing a block of porous material of rectangular cross section, Fig. 1. The input plane of the waveguide is excited in the dominant $T E_{10}$ mode having the spacial distribution corresponding to this mode and with an amplitude of $100 \frac{\mathrm{kV}}{\mathrm{m}}$. The excitation plane is located far away from the junction where the higher order modes are virtually non-existent. The energy which is reflected back due to a mismatch between the impedance of the waveguide and that of the located cavity passes through the excitation plane, which appears transparent, and is absorbed by the absorbing boundary. 


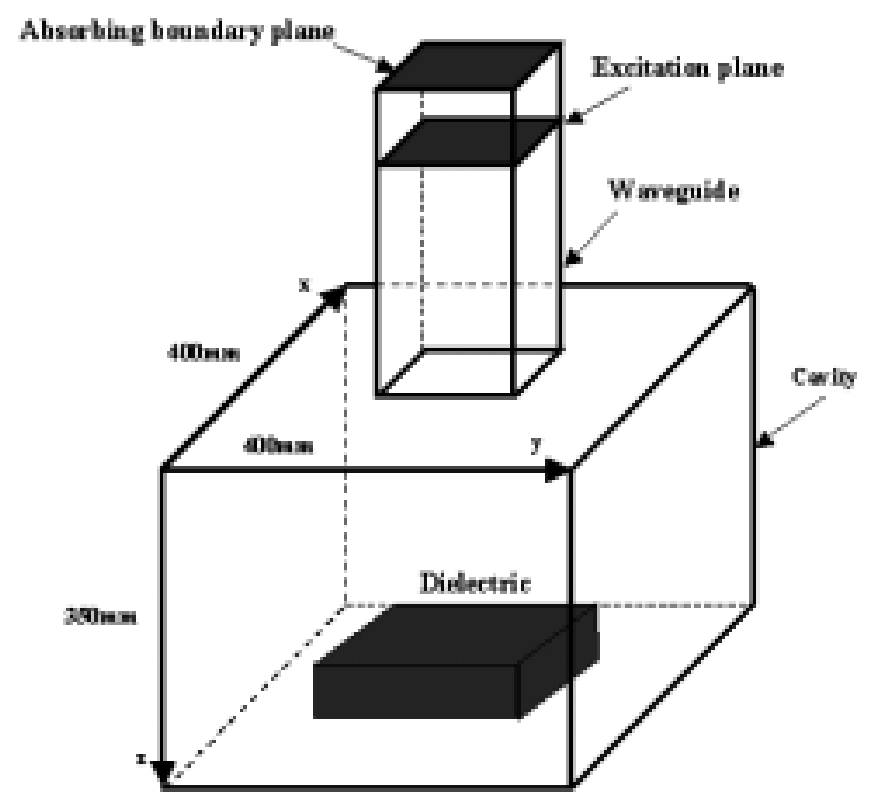

Fig. 1. Microwave oven model

\subsection{Solution Technique}

The coupling algorithm can be summarized as follows: the FTDT solver is run until the steady state (pure time harmonic) is sufficiently well approximated and the power distribution $Q$, computed from the peak field amplitudes, has converged. The temperature distribution and moisture content then evolve according to this power distribution until the electric properties of the medium have changed significantly. The electromagnetic solver is then re-run, taking into account the new electrical properties. Once the steady state is reached again, the whole procedure is repeated until the required heating time is reached.

It should be pointed out that the electromagnetic field distribution within the microwave oven is stabilized by the dielectric properties of the biomaterial on a timescale that is very small compared with the thermal process. There are many possible criteria that could be used to determine when the electromagnetic field components have converged. However the key quantity linking the two models is the microwave heating function $Q$. This heating function was determined at each FTDT time step and the total sum over the biomaterial calculated i.e.

$$
I^{n}=\int_{V} d V=\frac{1}{2} \sum_{i, j, k} \sigma_{i, j, k}\left|E_{i, j, k}^{\max }\right|^{2} \Delta x_{i} \Delta y_{j} \Delta z_{k} .
$$

The relative change in the total sum was used as a measure of the convergence of the FTDT scheme to the time-harmonic state. 
The cavity and the waveguide are meshed for the FTDT computation with a tensor-product cartesian mesh, but only the biomaterial needs to be meshed for the heat and mass transfer calculations within PHOENICS. Since the two meshes are independent, a transfer function (conserving the total $Q$ for the biomaterial) is used to map the heating function $Q$ from the FTDT mesh to the heat and mass transfer mesh. The time step for the electromagnetic solver is limited by the stability condition

$$
\Delta t<\frac{c}{\left(\frac{1}{\Delta x^{2}}+\frac{1}{\Delta y^{2}}+\frac{1}{\Delta z^{2}}\right)^{\frac{1}{2}}}
$$

where $c=(\epsilon \mu)^{-1 / 2}$ is the local electromagnetic wave speed. The time step was set to be $95 \%$ of the minimum time step for the mesh and material.

\subsection{Discussion}

Results are shown for a simple biomaterial with material properties set to those of mashed potato, with density $\rho=1050 \frac{\mathrm{kg}}{\mathrm{m}^{3}}$, and porosity $\phi=0.55$. The dielectric properties of mashed potato as a function of temperature at $10^{9} \mathrm{~Hz}$ were taken from literature [4]. Values for the liquid and gas permeabilities were $K_{l}=5 \times$ $10^{-16} m^{-2}$ and $K_{g}=1 \times 10^{-16} m^{-2}$, respectively. The initial temperature was taken to be $15^{\circ} \mathrm{C}$. An ambient temperature of $25^{\circ} \mathrm{C}$ was assumed together with a convective heat transfer of $10 \mathrm{Wm}^{-2} \mathrm{~K}^{-1}$ corresponding to a flow of air of approximately $0.5 \mathrm{~ms}^{-1}$ across the surface via the action of the oven fan. The slab had dimensions $200 \mathrm{~mm} \times 200 \mathrm{~mm} \times 4 \mathrm{~mm}$ and was positioned such that the planes of the horizontal and vertical symmetry of the load coincided with those of the waveguide. The load was placed at a distance of $17 \mathrm{~mm}$ from the oven-waveguide junction. The total heating time was $120 \mathrm{~s}$.

Due to the symmetry of the oven, the maximum values of the heating function on the face are in the centre, Fig. 2. The calculated temperature distributions is shown in Fig. 4. Temperature increase will be very steep in the centre corresponding to the power distribution, slowing down towards the edges due to surface cooling.

Microwave heating generates heat constantly, and as the temperature increases a change of phase will occur and the moisture content will decrease accordingly, Fig. 5. The elevated internal temperature and increasing internal vapor pressure drive the liquid from the medium quickly and efficiently. The vectors in Fig. 5 show the transfer of fluid out of the potato due to evaporation. Further increase of the temperature will further decrease liquid concentrations and vapour transport will become the dominant migration mechanism.

As the water in the food is transferred into vapour and is lost through the boundary, energy absorption is reduced because liquid water is the most active component in absorbing microwave energy. The microwave power distribution will change as shown in Fig. 3 . 

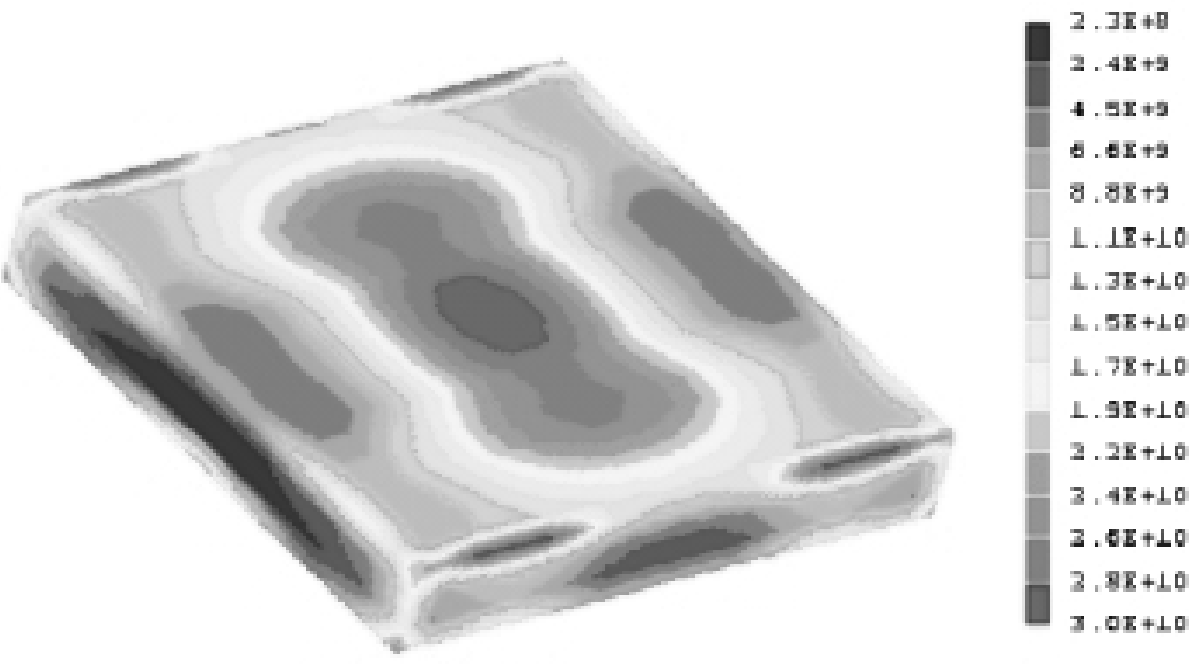

Fig. 2. Heating function, $Q$, inside the potato at $t=20 \mathrm{sec}$

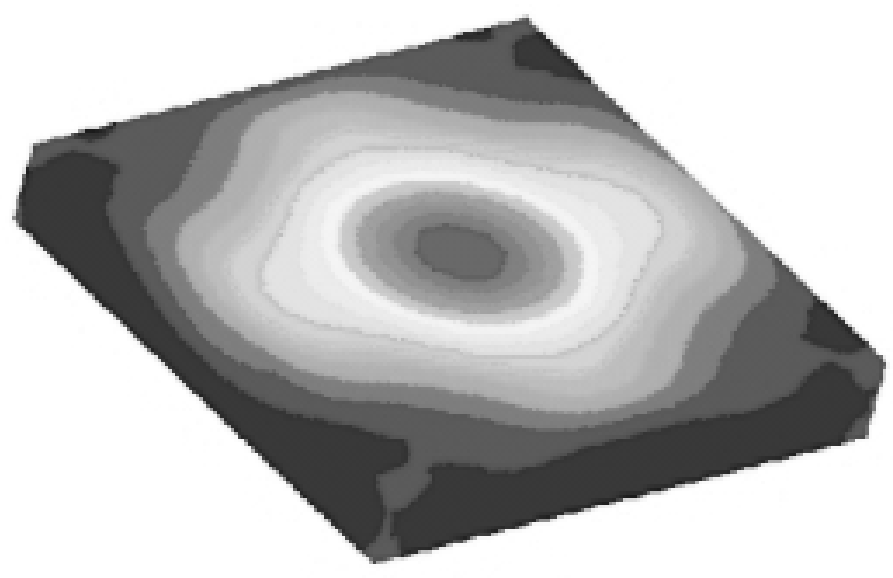

सrI

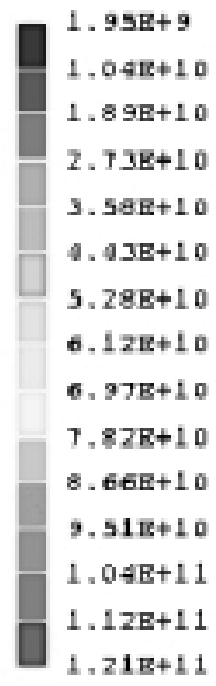

Fig. 3. Heating function, $Q$, inside the potato at $t=120 \mathrm{sec}$ 


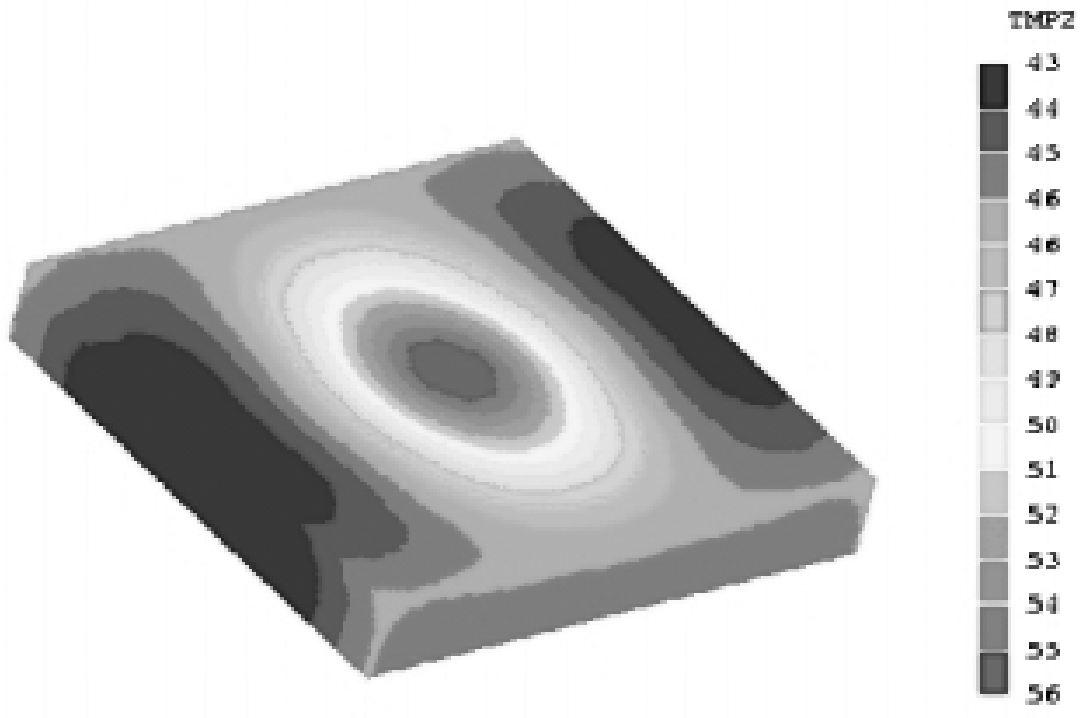

Fig. 4. Temperature profile inside the potato at $t=120 \mathrm{sec}$
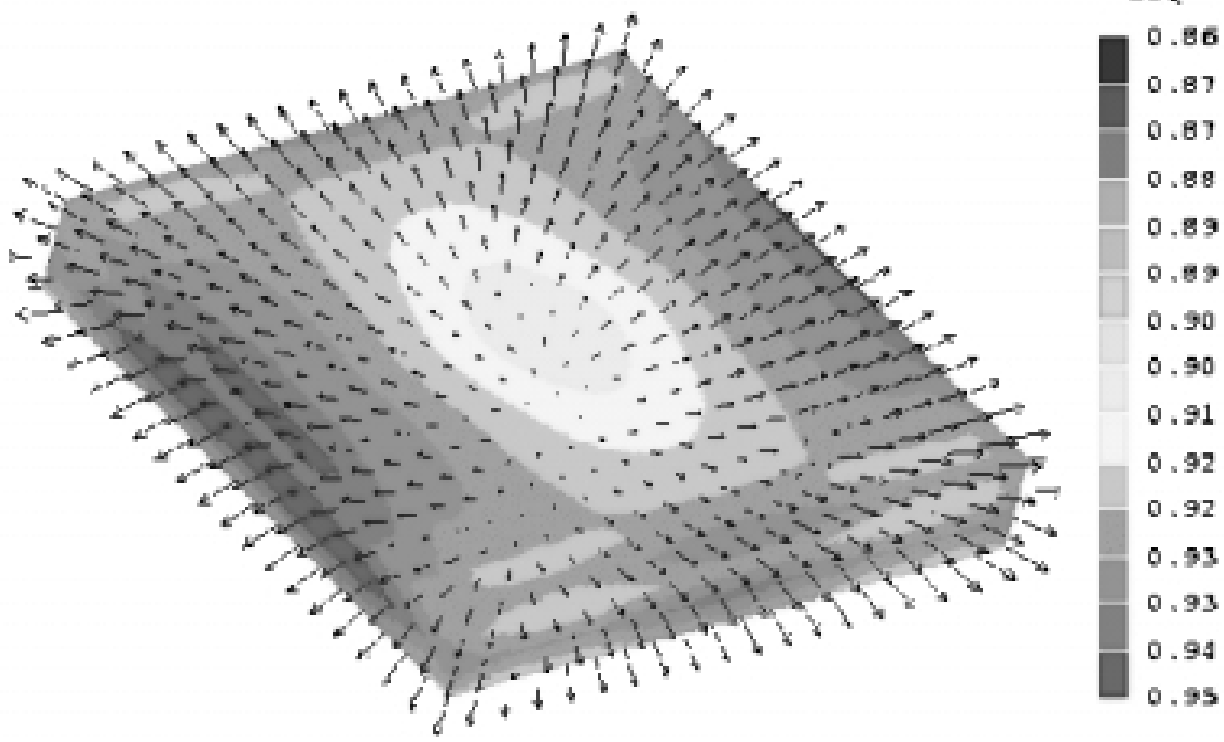

Fig. 5. Liquid concentration inside the potato at $t=40 \mathrm{sec}$ 


\section{Conclusion}

Heat and mass processes during the microwave heating of food materials are complicated and require knowledge of several areas. The model, which combines a three-dimensional transfer code with a three-dimensional electromagnetic computational scheme, was able to predict the overall drying behaviour. The two calculations have been closely coupled in an optimally cost-efficient manner. This model can be used as a tool for studying in detail microwave heating for real cases, provided the exact dependencies of dielectric properties are measured and implemented in the code.

\section{References}

1. Ayappa, K.G., Davis, H.T., Davis, E.A.,Gordon J.: Analysis of Microwave Heating of Materials with Temperature-Dependent Properties. AEChE Journal 37 (1991) 313-322

2. Bear, J.: Dynamics of Fluids in Porous Media. Dover Publications, New York (1998)

3. Jia, X.,Jolly,P.: Simulation of microwave field and power distribution in a cavity by a three dimensional finite element method. Journal of Microwave Power and Electromagnetic Energy 27 (1992) 11-22

4. Kent,M.: Electrical and Dielectric Properties of Food Materials. Science and Technology Publishers Ltd, England,(1987)

5. Metaxas, A.C., Meredith, R.J.: Industrial Microwave Heating. IEE Power Engineering Series, 4 (1983)

6. Monk, P.: Sub-Gridding FTDT Schemes. ACES Journal 11 (1996) 37-46

7. Monk, P., Suli, E.: Error estimates for Yee's method on non-uniform grids. IEEE Transactions on Microwave Theory and Techniques 30 (1994)

8. Ni, H., Datta, A.K., Torrance,K.E.: Moisture transport in intensive microwave heating of biomaterials: a multiphase porous media model. Int. J. of Heat and Mass Transfer 42 (1999) 1501-1512

9. Perre, P., Turner, I.W.: A 3-D version of TransPore: a comprehensive heat and mass transfer computational model for simulating the drying of porous media. Int. J. of Heat and Mass Transfer 42 (1999) 4501-4521

10. PHOENICS code,CHAM ltd, Wimbledon (http://www.cham.co.uk)

11. Torres, F., Jecko,B.: Complete FTDT Analysis of Microwave Heating Process in Frequency-Dependent and Temperature-Dependent Media. IEEE Transactions on Microwave Theory and Techniques 45 (1997) 108-117

12. Turner, I.,Jolly,P..: The effect of dielectric properties on microwave drying kinetics. Journal of Microwave Power and Electromagnetic Energy 25 (1990) 211-223

13. Yee, K.S.: Numerical solution of initial boundary value problems involving Maxwell's equations in isotropic media. IEEE Trans. Antennas Propag., 14 (1996) 302-307

14. Zhao, L., Puri,V.M., Anantheswaran,G., Yeh,G.: Finite Element Modelling of Heat and Mass Transfer in Food Materials During Microwave Heating-Model Development and Validation. Journal of Food Engineering 25 (1995) 509-529 